\title{
PADRÃO DE SUPERVISÃO DA ENFERMEIRA EM HOSPITAIS DE FEIRA DE SANTANA - BA '
}

NURSE SUPERUISION PATTERN AT HOSPITALS IN FEIRA DE SANTANA - BA.

\author{
Maria Lúcia Servo Leite ${ }^{2}$
}

\begin{abstract}
RESUMO: Estudo exploratório descritivo sobre Padrão de Supervisão da Enfermeira (P.S.E.), realizado em Feira de Santana-Bahia-Brasil, em 1994, tendo como objetivos descrever o padrão de supervisão e identificar os fatores que interferem na mesma. Utilizou-se questionário e estatística descritiva. O padrão de supervisão realizado pela enfermeira não é sistematizado, evidenciando a falta de percepção da supervisão enquanto processo. Estatisticamente comprovou-se que o tipo de hospital é um fator que interfere no P.S.E. Respaldada na literatura, no exame percentual dos resultados e na experiência profissional da autora, afirma-se que sexo, formação acadêmica, remuneração, política de saúde, planejamento e estrutura técnico administrativa são fatores condicionantes do P.S.E., assim como, mencionados pelos sujeitos, a falta de autonomia profissional, as interferências políticas partidárias, deficiência de recursos humanos, baixa remuneração, deficiência de recursos materiais, ambiente físico inadequado e relações interpessoais insatisfatórias.
\end{abstract}

UNITERMOS: Supervisão sistematizada da enfermeira. Supervisão em enfermagem. Qualidade da intervenção da enfermeira.

\begin{abstract}
This is an exploratory descriptive study about Nurse Supervision Pattern (NSP) performed in Feira de Santana-Bahia-Brazil, in 1994, which aims at describing supervision pattern and identifying interference factors. A questionnaire and descriptive statistics have been used. Supervision pattern proved that the kind of hospital is a factor that interferes on NSP. Based on literature, percentual exam of results and author's professional experience, it asserted that sex, academic graduation, earnings, health politics, planning and technical administrative structure are conditional factors for NSP, as well as lack of professional autonomy, interference of political parties, deficiency of material and human resources, low salaries, unsuitable environment and unsatisfactory interpersonal relationships mentioned by the subjects.
\end{abstract}

KEYWORDS: Nurse systematic supervision - Nursing supervision - Nursing intervention quiality.

\footnotetext{
Trabalho apresentado no $48^{\circ}$ Congresso Brasileiro de Enfermagem, ocorrido no período de 6 a 11 de outubro de 1996, em são Paulo - SP.

Enfermeira. Doutoranda em Enfermagem da Escola de Enfermagem da Universidade de São

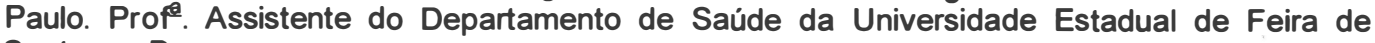
Santana - Ba.
} 


\section{INTRODUÇÃO}

O Setor Saúde, no Brasil, passa por um momento histórico caracterizado pelo modelo assistencial preconizado pelo Sistema Único de Saúde (SUS) criado pela Constituição Federal ${ }^{3}$ e regulamentado pela Lei Orgânica de Saúde no $8080 / 90^{9}$, Lei $8.142 / 90$ e Decreto $99.438 / 908$. Na implantação deste modelo assistencial, os recursos humanos são o eixo fundamental para a eficácia do Sistema.

A intervenção da enfermeira ${ }^{3}$ contribui para a integralidade da atenção à saúde, que é um direito do cidadão, e a sua prática, portanto, deve ser analisada e contextualizada, de, maneira que seja capaz de acompanhar e inserir-se no processo de mudanças econômico-sócio-culturais e políticas.

O presente estudo foi gerado das reflexões que surgiram em decorrência da nossa experiência tanto na área assistencial como na coordenação do Serviço de Enfermagem, em um Hospital Geral do Estado, no município de Feira de Santana-Bahia, durante o período de 1983-1988, e também da experiência acumulada ao longo de 7 anos como docente da disciplina Administração Aplicada à Enfermagem ${ }^{4}$, quando se questionava como a supervisão havia sido feita? como deveria ser? qual o seu real significado? qual a percepção das enfermeiras sobre a supervisão? em que base a supervisão está inserida no processo de trabalho da enfermeira? a supervisão, enquanto função inerente da enfermeira, é realizada de forma sistematizada ou não sistematizada? e qual o padrão de supervisão existente?

Neste estudo o padrão de supervisão constitui-se em supervisão sistematizada e não sistematizada. O padrão de supervisão sistematizada deve ser compreendido como um processo que envolve planejamento, execução e avaliação das atividades realizadas, através da utilização de técnicas e instrumentos de supervisão que visam aferir eficiência, eficácia e efetividade, proporcionando o desenvolvimento da capacidade individual, grupal e de relacionamento interpessoal da equipe de enfermagem e qualidade da intervenção prestada aos usuários do sistema de saúde. É importante destacar que dentro da concepção de padrão de supervisão sistematizada proposta neste estudo, a eficiência refere-se à forma de realizar a tarefa em conformidade com o que foi planejado mediante padrões preestabelecidos, estando associada à quantidade, à produtividade e aos custos; a eficácia traduz o resultado da tarefa, isto é, a adequação do produto para os fins a que se propõe, estando associada

\footnotetext{
3 Utilizar-se-á o gênero feminino quando nos referimos às enfermeiras e às supervisoras, buscando destacar a alta freqüência de mulheres nesta categoria profissional $(94,1 \%$ no Brasil, em 1983, COFEN/ABEn p.57 vol. I).

Esta disciplina faz parte do ciclo de disciplinas profissionalizantes do Curso de Enfermagem do Departamento de Saúde da Universidade Estadual de Feira de Santana, oferecida no $8^{\circ}$ semestre.
} 
aos-objetivos, à qualidade e à satisfação dos usuários; e a efetividade é a interação da conformidade (eficiência) com a adequação (eficácia), ou seja, é fazer a coisa certa da forma certa - é otimizar a supervisão em forma de processo.

A supervisão realizada pela enfermeira é entendida como função inerente à sua prática, independente do cargo/função ${ }^{5}$ que esteja exercendo, pois, legalmente e no exercício profissional, esta é uma supervisora em potencial.

O Decreto-Lei no 94.406 , de 08 de junho de $1987^{7}$ que regulamenta a Lei 7.498 de 25 de junho de $1986^{6}$, que dispõe sobre o exercício de enfermagem no Brasil, em seu artigo 80 diz que ao enfermeiro incumbe privativamente, na alínea c, "o planejamento, organização, execução e avaliação dos serviços de assistência de enfermagem" e nas atividades como integrante da equipe de saúde, ressalta-se a "participação na elaboração de medidas de prevenção e controle sistemático de danos que possam ser causados aos pacientes durante a assistência de enfermagem" (alínea f); "participação em programas e atividades de educação sanitária, visando à melhoria de saúde do indivíduo, da família e da população em geral" (alínea $\mathrm{m}$ ); e "participação nos programas de treinamento e aprimoramento de pessoal de saúde, particularmente nos programas de educação continuada" (alínea n) (p. 25-26).

Com base nestas reflexões, formulou-se o seguinte questionamento:

\section{- Qual o padrão de supervisão realizada pela enfermeira em hospitais de Feira de Santana-Ba, no período de março a junho de 1994? \\ Objetivos}

1 - Descrever o padrão de supervisão executada pela enfermeira da área hospitalar.

2 - Identificar os fatores que interferem no padrão de supervisão realizada pela enfermeira da área hospitalar.

\section{Hipóteses}

$\mathrm{H}_{1}$ - O padrão de supervisão executada pela enfermeira que atua na área hospitalar não é sistematizada.

$\mathrm{H}_{2}$ - $\mathrm{O}$ padrão de supervisão executada pela enfermeira sofre interferência de fatores associados ao perfil da enfermeira e às características das instituições hospitalares.

Para MEZOMO, Função é um conjunto de tarefas executadas de maneira sistemática por uma ou várias pessoas e Cargo é o posicionamento hierárquico na estrutura orgânica da empresa. (MEZOMO, João C. - Administração de Recursos Humanos. São Paulo: CEDAS, 1984, p. 66). 


\section{PROCEDIMENTOS METODOLÓGICOS}

\section{Modelo do estudo}

Para alcançar os objetivos propostos, o modelo da investigação é classificado como um estudo transversal exploratório-descritivo (nivel 1), segundo Wilson ${ }^{21}$, Richardson ${ }^{19}$ e Gil ${ }^{15,16}$.

\section{Universo e população}

Identificou-se quatorze (14) hospitais em Feira de Santana-Ba., cadastrados pela Associação dos Hospitais do Estado da Bahia. Destes, cinco (05) não dispunham de enfermeiras em seu quadro de pessoal.

Pela natureza do trabalho, estabeleceu-se que o estudo seria realizado em instituições hospitalares que dispusessem de enfermeiras em seu quadro de pessoal e que estivessem em pleno exercicio profissional no local de pesquisa.

Desta forma, o universo deste estudo, que foi realizado no período de março a junho de 1994, constituiu-se de nove (09) hospitais de Feira de Santana-Ba, ficando, portanto, a população de estudo constituída de oitenta e três (83) enfermeiras, o que corresponde a $81,37 \%$ da população de enfermeiras existentes nos hospitais estudados. Contudo, a homogeneidade do grupo estudado - enfermeiras da área hospitalar - nos garante a representatividade dos dados analisados.

\section{Escolha da técnica para coleta de dados}

A técnica escolhida para a realização do levantamento dos dados foi o questionário, respondido pelas enfermeiras.

\section{0 instrumento}

O questionário foi elaborado e estruturado com questões abertas e fechadas, com alternativas dicotômicas, tricotômicas, respostas múltiplas e abertas, de modo que fosse possivel atingir os objetivos traçados para o estudo.

O instrumento compõe-se de duas (02) partes assim constituídas:

1a parte: $A$ enfermeira e as instituições hospitalares

A - Perfil da clientela estudada

B - Características da instituição.

2a parte: A enfermeira e a aplicação do padrão de supervisão

C - Planejamento

D - Execução

E - Avaliação. 
A 1a parte do instrumento totaliza 16 questões, cujos dados permitiram a análise das variáveis independentes estabelecidas neste estudo, e a 2 a parte do instrumento totaliza 17 questões. Esses dados permitiram a análise da variável dependente estabelecida para este estudo - padrão de supervisão.

\section{Aplicação e procedimentos utilizados na coleta de dados}

Realizou-se um estudo piloto aplicando o instrumento a quinze (15) enfermeiras de hospitais da cidade de Salvador-Ba., com o objetivo de verificar sua adequação ao estudo, bem como ajustar as falhas e eliminar respostas preelaboradas.

Visitou-se as instituições hospitalares e contatou-se com os diretores, chefias de enfermagem e enfermeiras para fins de agendamento do período de entrega do questionário pessoalmente a cada enfermeira e recebimento dos questionários em cada instituição.

\section{Processamento, tratamento estatístico e análise dos resultados}

Utilizou-se procedimentos de estatística descritiva. Quanto à decodificação e categorização das questões abertas, procurou-se utilizar o critério semântico, observando as características de exaustividade, exclusividade, homogeneidade, objetividade e fidelidade.

A variável dependente Padrão de Supervisão foi analisada mediante valores e pesos atribuídos ao planejamento, execução e avaliação. Estabeleceu-se a seguinte classificação: Planejamento (08 pontos e peso 05 ) totalizando 40 pontos; Execução (22 pontos e peso 02 ) totalizando 44 pontos; Avaliação (18 pontos e peso 02 ) e ainda o item Satisfação (02 pontos e peso 01$)$ totalizando 38 pontos. Esta variável somou 122 pontos.

A classificação ora apresentada foi utilizada visando o estabelecimento do padrão de qualidade deste estudo para designar supervisão sistematizada e não sistematizada.

O padrão de supervisão sistematizada foi obtida mediante as incidências de respostas "sim" extraídas do instrumento de coleta de dados, representando a aplicação plena da supervisão entendida como processo, indicando a operacionalidade da sistematização da supervisão. Estabeleceu-se a média igual ou acima de $60 \%$ para o padrão de supervisão sistematizada.

Quanto às incidências de respostas "às vezes" e "não", evidenciou-se as aplicações eventuais da supervisão como processo ou ausência de otimização deste processo; ambas sintetizam as oscilações da supervisão em estudo. Neste nivel, caracterizou-se o padrão de supervisão como não sistematizada, cuja média obtida permaneceu abaixo de $60 \%$.

Desta forma, computou-se a soma dos pontos de cada questionário referente ao somatório das respostas da variável padrão de supervisão - planejamento, execução e avaliação e dividiu-se pelo total máximo de pontos que cada 
respondente deveria atingir, obtendo-se a média. Diante da média obtida e da estabelecida para o estudo enquadrou-se em padrão de supervisão sistematizada ou não sistematizada.

Assim, os resultados obtidos mediante aplicação do instrumento foram computados e confrontados, segundo o padrão de supervisão sistematizada e padrão de supervisão não sistematizada, englobando diversos tipos de informações através da associação entre as variáveis independentes com a aplicação do teste de $X^{2}$ (Qui-quadrado) de Pearson e $X^{2}$ de Fischer e desvio padrão.

Os dados obtidos foram registrados em tabelas simples e compostas.

A partir das informações constantes no questionário, procedeu-se à análise quantitativa com o aporte da abordagem qualitativa, obedecendo as unidades temáticas estabelecidas quando da delimitação das variáveis de estudo.

Richardson ${ }^{19}$, referindo-se ao aporte do método qualitativo ao quantitativo, afirma que na análise da informação, as técnicas qualitativas permitem verificar os resultados dos questionários e ampliar as relações descobertas (p.48), ou seja, permitem apreender o conhecimento e a intimidade, com o objeto de estudo. Este autor, assinala ainda que o método qualitativo difere, em princípio, do quantitativo à medida que não emprega um instrumental estatístico como base do processo de análise de um problema e comenta que Há autores que não fazem distinção clara entre métodos quantitativos e qualitativos, por entender que a pesquisa quantitativa é também de certo modo, qualitativa ( $p$. 38).

Neste estudo procedeu-se com a análise quantitativa utilizando como aporte a abordagem qualitativa para a realização do mesmo e assim reportou-se às citações dos atores sociais que participaram deste estudo, visando apreender e valorizar os dados objetivos e subjetivos interiorizados e manifestados. Tais citações são procedidas de um número que corresponde ao número do questionário.

Prossegue-se com a apresentação, análise e discussão dos resultados.

\section{APRESENTAÇÃO, ANÁLISE E DISCUSSÃO DOS RESULTADOS}

\section{Apresentação e análise dos dados}

Far-se-á a leitura das tabelas, levando em consideração apenas os dados que forem mais relevantes para este estudo.

A Tabela 01, mostra a distribuição do padrão de supervisão encontrado e que é desenvolvido pela enfermeira nas instituições hospitalares estudadas. 
TABELA 1- PADRÃO DE SUPERVISÃO DA ENFERMEIRA EM HOSPITAIS.

FEIRA DE SANTANA-BA, 1994.

\begin{tabular}{l|c|c}
\hline \multirow{2}{*}{ PADRÃO DE SUPERVISÃO } & \multicolumn{2}{|c}{ FREQÜÉNCIA } \\
\cline { 2 - 3 } & $\mathbf{F i}$ & $\%$ \\
\hline Sistematizada & 03 & 3,61 \\
\hline Não Sistematizada & 80 & 96,39 \\
\hline TOTAL & $\mathbf{8 3}$ & $\mathbf{1 0 0 , 0 0}$ \\
\hline
\end{tabular}

Conforme nos mostra a Tabela acima, o padrão de supervisão não sistematizada alcançou freqüência superior correspondendo a 96,39\% do total da população, seguido do padrão de supervisão sistematizada com indice percentual de $3,61 \%$.

Assim, o primeiro grau (sistematizada) englobou as atividades de supervisão realizadas sob forma de processo (planejamento, execução e avaliação) e o segundo grau (não sistematizada) é relativo às atividades que são realizadas em forma de processo eventualmente ou não.

As tabelaṣ a seguir demonstram a relação entre o padrão de supervisão e o perfil da clientela estudada.

TABELA 02 - PADRÃO DE SUPERVISÃO DA ENFERMEIRA SEGUNDO O TIPO DE HOSPITAL. FEIRA DE SANTANA-BA, 1994

\begin{tabular}{l|c|c|c|c|c|c}
\hline $\begin{array}{l}\text { PADRÃO DE } \\
\text { SUPERVISÃO }\end{array}$ & \multicolumn{2}{|c|}{ SISTEMATIZADA } & \multicolumn{2}{|c}{$\begin{array}{c}\text { NÃO } \\
\text { SISTEMATIZADA }\end{array}$} & \multicolumn{2}{c}{ TOTAL } \\
\hline TIPO DE HOSPITAL & $\mathrm{Fi}$ & $\%$ & $\mathrm{Fi}$ & $\%$ & $\mathrm{Fi}$ & $\%$ \\
\hline Geral & - & - & 57 & 68,68 & 57 & 68,68 \\
\hline Especializado & 03 & 3,61 & 23 & 27,71 & 26 & 31,32 \\
\hline TOTAL & $\mathbf{0 3}$ & $\mathbf{3 , 6 1}$ & $\mathbf{8 0}$ & $\mathbf{9 6 , 3 9}$ & $\mathbf{8 3}$ & $\mathbf{1 0 0 , 0 0}$ \\
\hline $\mathrm{x}^{2}=6,82 ; \mathrm{p}=0,00899632 ; \mathrm{x}^{2}$ Fischer $= \pm 0,0282975$
\end{tabular}

Verificou-se que $68,68 \%$ das respondentes trabalham em hospital geral e realizam supervisão não sistematizada. Constatou-se que 3,61\% atuam em hospitais especializados e sistematizam a supervisão. Observou-se que estatisticamente existe associação entre padrão de supervisão e tipo de hospital $\left(X^{2}\right.$ Fischer $\left.\pm 0,0282975\right)$ em nivel de $5 \%$ de probabilidade.

\section{Discussão dos Resultados}

\section{A enfermeira e a aplicação do padrão de supervisão}

A Enfermagem compõe o grupo que faz parte da atenção à saúde da população e a supervisão em enfermagem é apontada por diversos autores como um instrumento que viabiliza a qualidade da intervenção de enfermagem, 
pois procura desenvolver as potencialidades dos membros da equipe no sentido de proporcionar uma assistência adequada e livre de riscos aos usuários do sistema de saúde. Observadas as especificidades, os documentos Padrões Minimos de Assistência de Enfermagem à Comunidade ${ }^{4}$, Padrões Minimos da Assistência de Enfermagem em Recuperação da Saúde ${ }^{5}$, a Lei do Exercício Profissional da Enfermagem ${ }^{6}$, o Código de Defesa do Consumidor ${ }^{10}$ e o Código de Ética dos Profissionais de Enfermagem ${ }^{12}$ vislumbram estes aspectos.

Para se ter uma supervisão eficiente, é necessário a adoção de uma metodologia de trabalho que a contemple como processo dinâmico que envolve o planejamento, a execução e a avaliação das atividades realizadas, visando detectar os pontos de estrangulamento e buscar os métodos e técnicas mais eficazes na continuidade da assistência aos clientes e na satisfação pessoal daqueles que trabalham nesta assistência. Portanto, é imprescindivel, para se otimizar o trabalho, a utilização sistemática dos métodos, técnicas e instrumentos de supervisão pela enfermeira.

O caminho percorrido para se chegar ao padrão de supervisão nos permitiu a análise do planejamento, execução e avaliação.

Verificou-se a operacionalização do planejamento e obteve-se os dados a seguir: $69,9 \%$ da enfermeiras referiram existir plano de trabalho no serviço de enfermagem, porém, $68,7 \%$ não sistematizam o planejamento da assistência aos clientes; $55,4 \%$ não planejam as atividades diárias e $54,2 \%$ não fazem plano individual de trabalho.

Diante do exposto, afirma-se que a existência de Plano de Trabalho no Serviço de Enfermagem nos hospitais estudados, segundo $69,9 \%$ dos sujeitos, é um indicativo de que as decisões tomadas terão menos margem de erro e de perdas, facilitando a sistematização e análise da situação. No entanto, $68,68 \%$ não sistematizam o planejamento da assistência e $31,32 \%$ das enfermeiras fazem a, sistematização do planejamento da assistência aos clientes, mostrando uma incoerência da prática na operacionalização do processo de planejamento.

Concorda-se com Castilho e Gaidzinski" quando afirmam que "o requisito fundamental para que seja mudada a forma de assistir a clientela é a conscientização do enfermeiro em relação à importância e a necessidade do uso de um método de sistematização da assistência (p.210). " Portanto, constatou-se que $68,68 \%$ das enfermeiras não utilizam um método de trabalho para atender as necessidades da clientela, e assim não determinam as ações de enfermagem e conseqüentemente não utilizam a metodologia geral do planejamento. Concluiu-se que estes sujeitos atuam na amplitude operacional do planejamento, constituindo-se num percentual baixo da população.

A prioridade mais observada ao planejamento diário da atividade de supervisão é o número de pacientes e grau de dependência, referida por $37,3 \%$ dos sujeitos. 
A atividade de supervisão mais realizada pela enfermeira é a orientação e avaliação dos funcionários durante a execução das atividades para identificar necessidades de treinamento, foi referida por $67,5 \%$ dos sujeitos.

Para o Ministério da Saúde ${ }^{1,2}$ e Cunha $^{14}$, as técnicas de supervisão são: observação direta, revisão de documentos, avaliação de dados, entrevista, informação, demonstração de procedimentos, orientação, discussão de grupo, reunião e estudos especiais.

Cunha ${ }^{14}$ assinala que são inúmeras as técnicas que visam otimizar as ações dos grupos em seu ambiente de trabalho, constituindo-se em estratégias que desenvolvem os individuos, propiciam o alcance de maturidade profissional e facilitam a participação destes indivíduos no processo decisório, promovendo interação harmoniosa, produtiva e satisfatória.

Este estudo revelou que as enfermeiras utilizam várias técnicas de supervisão, sendo a reunião com os membros da equipe de enfermagem a mais realizada $(97,6 \%)$.

Os instrumentos de supervisão variam de acordo com os tipos de comunicação segundo Takahashiro. Cunha ${ }^{14}$ e documentos do Ministério da Saúde $^{1,2}$, assinalam que estes se constituem em: roteiros, dados estatísticos, relatórios de serviços, fichas e notas de pacientes, manuais de técnicas, normas, rotinas e procedimentos, mapas, comentários, fichas de avaliação e acompanhamento, inclusive objetivos e indicadores.

Este estudo revelou que o relatório é o instrumento de supervisão mais utilizado $(77,1 \%)$ e os roteiros em seguida $(57,8 \%)$.

Dada a importância da avaliação no processo de supervisão, visando a (re)elaboração de um planejamento efetivo e a execução das atividades planejadas, inquiriu-se os sujeitos desta pesquisa sobre os seguintes itens: aspectos observados na avaliação da assistência do cliente; avaliação ao trabalho da enfermeira; avaliação da instituição frente às dificuldades existentes que limitam o trabalho de supervisão da enfermeira e finalmente a satisfação da enfermeira com o trabalho que realiza na instituição.

Inquiridos sobre quais aspectos são observados na avaliação da assistência aos clientes, verificou-se que $51,8 \%$ dos sujeitos mencionaram a observação do prontuário, relatório e livro de ocorrência. Estes dados nos levaram ao seguinte questionamento: Como avaliar a assistência aos clientes se 68,68\% das enfermeiras não fazem a sistematização do planejamento da assistência aos clientes? Sabe-se que a avaliação é uma fase imprescindivel para a elaboração do planejamento, seja qual for a sua natureza: Será que as enfermeiras realmente planejam e avaliam? Ou será que executam sem avaliar e planejar?

O trabalho da enfermeira é avaliado através de relatórios, registros de ocorrências e prontuários (37,3\%). 
Com relação à avaliação da instituição frente às dificuldades existente"s que limitam o trabalho de supervisão da enfermeira, observou-se os seguintes dados: Falta de autonomia profissional( $80,7 \%)$ : Interferência política partidária e ingerência administrativa $(71,1 \%)$; Deficiência de recursos humanos quantiqualitativa (67,5\%); Deficiência de recursos materiais e ambiente físico inadequado (63,9\%); Baixa remuneração(55,4\%) e Relações interpessoais insatisfatórias(54,2\%).

Cunha ${ }^{14}$ assinala que existem situações que dificultam a supervisão, tais como: filosofia de enfermagem que não enfatiza a importância do desenvolvimento de pessoal e da manutenção de relações interpessoais; existência de uma política de trabalho centralizadora, autoritária e tarefista; inadequação de recursos humanos, materiais, físicos e financeiros; área física inadequada; o despreparo das enfermeiras para desenvolver a supervisão e falha no planejamento do serviço ou divisão de enfermagem.

Por outro lado, o MINISTÉRIO DA SAÚDE ${ }^{2}$ menciona que os problemas e dificuldades observadas no processo de supervisão referem-se à estrutura técnico-administrativa e de execução; transgressão dos procedimentos de trabalho; atitudes impróprias e infraestrutura.

Observou-se que os dados obtidos neste estudo, relacionados às dificuldades que as enfermeiras encontram para desenvolver o seu trabalho na instituição, condizem com o que prescreve a literatura. No entanto, não se encontrou referências à interferência política partidária mencionadas por $71,1 \%$ dos sujeitos deste estudo.

Mediante os resultados apresentados e aplicando o procedimento estatístico estabelecido para a caracterização do padrão de supervisão executado pelas enfermeiras da área hospitalar, identificou-se que $3,61 \%$ das enfermeiras atingiram a média maior ou igual a $0,60 \%$, ou seja, 6,0 pontos.

Desta forma, evidenciou-se a comprovação da primeira hipótese deste trabalho de que o padrão de supervisão executada pela enfermeira que atua na área hospitalar não é sistematizada(96,39\%), conforme Tabela 01.

\section{A enfermeira e as instituições hospitalares}

\section{O perfil da clientela estudada e a instituição em que trabalha}

Do total de enfermeiras que constituem a população deste estudo, $68,68 \%$ atuam em hospitais gerais e realizam supervisão não sistematizada; e 31,32\% atuam em hospitais especializados, e, $27,71 \%$ não sistematizam a supervisão enquanto que $3,61 \%$ fazem a sistematização. Estatisticamente existe associação significativa entre padrão de supervisão e tipo de hospital. 
Não há associação estatística significativa entre padrão de supervisão e as variáveis sexo, formação acadêmica, cargo, nível salarial, política de saúde, entidade mantenedora, capacidade operacional, clientela e número de enfermeiras. Estes resultados contrariam a literatura consultada que prescreve o planejamento como fator condicionante para o exercicio de uma supervisão efetiva, e estas variáveis são aspectos que devem ser observados para a realização de um planejamento compatível com as necessidades dos usuários e dos serviços (MINISTÉRIO DA SAÚDE ${ }^{12}$ ).

\section{CONSIDERAÇÕES FINAIS E SUGESTÕES}

\section{A enfermeira e a aplicação do padrão de supervisão}

As enfermeiras estão aplicando de modo aleatório os conhecimentos científicos quando na utilização de forma fragmentada e desarticulada dos métodos, técnicas e instrumentos de supervisão, ou então, operacionalizando o serviço sem associar a teoria com a prática; sinalizando um despreparo para o exercício da função e falha no planejamento do serviço de enfermagem em todos os níveis, evidenciando a falta de percepção da supervisão enquanto processo.

Apesar de encontrar-se predominância do padrão de supervisão não sistematizado (96,39\%), não se pode afirmar haver o exercício só desse padrão, estando as bases da supervisão e seu exercício na dependência de situações específicas da prática.

A falta de autonomia profissional $(80,7 \%)$, interferência política partidária e ingerência administrativa $(71,1 \%)$, deficiência de recursos humanos quantiqualitativa $(67,5 \%)$, deficiência de recursos materiais e ambiente físico inadequado $(63,9 \%)$, baixa remuneração $(55,4 \%)$, relações interpessoais insatisfatórias $(54,2 \%)$ e insatisfação das enfermeiras com o trabalho que realizam $(57,8 \%)$ são fatores limitantes do exercício da supervisão.

\section{A enfermeira e as instituições hospitalares}

Comprovou-se estatisticamente haver discordância significativa a nível de 5\% de probabilidade entre as variáveis categorizadas no perfil da enfermeira e características da instituição com o padrão de supervisão, exceto a variável tipo de hospital. Entretanto, com exceção da variável idade, tempo de formada e outro vínculo empregatício, observa-se a relevância que as demais variáveis assumem quando do exame percentual e da vivência profissional da autora e verifica-se a importância que cada uma representa para o padrão de supervisão da enfermeira, pois refletem a qualificação đo individuo, política de trabalho, recursos humanos, remuneração, planejamento e estrutura técnicoadministrativa, aspectos referenciados como fatores condicionantes para o êxito da supervisão pela literatura. 
Contudo, o resultado exige postura de investigação com base na metodologia científica. Considerando os resultados alcançados, ressalta-se que a realidade da prática representa a tendência do grupo a um estágio de sensibilização para atender ou não às necessidades da profissão.

Corrobora-se o pensamento de Minayo $^{18}$ :

O conhecimento é um processo infinito e não há condição de fechá-lo numa fase final, assim como não se pode fechar o final do processo histórico; embora o projetemos como politicamente democrático e socialmente igualitário para ser ecologicamente saudável. (p. 18)

\section{Sugestões}

\section{Às Enfermeiras}

Participem do levantamento das áreas prioritárias para a pesquisa em enfermagem e que os resultados desta sejam divulgados e os pesquisadores informem aos sujeitos e as Instituições os resultados obtidos nas investigações.

\section{Às Escolas de Enfermagem}

Realizem estudos sobre o nível de conscientização das enfermeiras a cerca das contradições que permeiam a sua prática e seus fatores determinantes;

\section{Às Entidades de Classe}

Mobilizem os profissionais de enfermagem para discussão ampla visando uma tomada de posição (em nível local, estadual e federal) para a construção de um código de conduta ou um guia para a administração, onde se visualize a dimensão ética dos gerentes, profissionais de saúde e usuários.

\section{REFERÊNCIAS BIBLIOGRÁFICAS}

1. BRASIL. Constituição, 1988. República Federativa do Brasil. Brasília: Senado Federal/Centro gráfico, 1988. p. 48.

2. Ministério da Saúde/OPAS/OMS. Padrões mínimos de assistência de enfermagem à comunidade. Informe Final. Brasilia: 1977, 71p.

3. Ministério da Saúde/OPAṠ/OMS. Padrões mínimos de assistência de enfermagem em recuperação da saúde. Informe Final. Brasilia: 1978. 66p. 
4. BRASIL. Ministério da Saúde/Secretaria Nacional de Ações Básicas de

Saúde. Contribuição ao Desenvolvimento do Processo de Supervisão. Brasília, Centro de Documentação do Ministério da Saúde. 1980. 59p.

5. Ministério da Saúde/Secretaria Nacional de Ações Básicas de Saúde. Guia de Supervisão em Estabelecimento de Saúde. Brasilia: Centro de Documentação do Ministério da Saúde, 1983. 25p.

6. Lei no 7.498 de 25 de junho de 1986. Lei do Exercício Profissional de Enfermagem. Diário Oficial da República Federativa do Brasil, Brasilia, 26 jun. 1986.

7. Decreto no 94.406 (Regulamentação da Lei do Exercício Profissional de Enfermagem) de 08 de junho de 1987. Diário Oficial da República Federativa do Brasil, v. 125, n. 106, p. 8853-8855, jun. 1987.

8. Decreto $\mathrm{n}$ ㄴ 99.438 que dispõe sobre a Organização e atribuições do Conselho Nacional de Saúde, de 07 de agosto de 1990. Diário Oficial da República Federativa do Brasil, Brasilia, 1990.

9. Lei Orgânica de Saúde no 8.080 de 19 de setembro de 1990. Diário Oficial da República Federativa do Brasil, Brasilia, 1990.

10. Lei no 8.078. Código de Defesa do Consumidor. Diário Oficial da República Federativa do Brasil, Brasília, 12 set. 1990.

11. CASTILHO, Valéria; GAIDZINSKI, Raquel Rapone. Planejamento da assistência de enfermagem. In: KURCGANT, Paulina (coord.) Administração em enfermagem. São Paulo: EPU, 1991. p. 207-214.

12. CONSELHO FEDERAL DE ENFERMAGEM. Código de ética dos profissionais de enfermagem. Resolução $\mathrm{n}$ ㅇ 160 de 12/05/93. Rio de Janeiro, 1993. 15p.

13. CONSELHO FEDERAL DE ENFERMAGEM / ASSOCIAÇÃO BRASILEIRA DE ENFERMAGEM. Força de trabalho em enfermagem: o exercício da enfermagem nas instituições de saúde do Brasil - 1982/1983. Rio de Janeiro. COFEn/ABEn, 1985. v. 1, 236p.

14. CUNHA, Kathia de Carvalho. Supervisão e enfermagem. In: KURCGANT, Paulina (coord.) Administração em enfermagem. São Paulo: EPU. 1991 p. 117 - 132. 
15. GIL, Antonio Carlos. Como elaborar projetos de pesquisa. 2. ed. São Paulo: Atlas, 1989. 159p.

16. Métodos e técnicas de pesquisa social. 3. ed. São Paulo: Atlas, 1991. 207p.

17. MEZOMO, João Catarin. Administração de recursos humanos. São Paulo: CEDAS, 1984. 233p.

18. MINAYO, Mária Cecília de Souza. O desafio do conhecimento: Pesquisa qualitativa em saúde 3. ed. São Paulo - Rio de Janeiro, HUCITEC-ABRASCO, 1994. 269p.

19. RICHARDSON, Roberto Jarry (col.). Pesquisa social: métodos e técnicas. 2. ed. São Paulo: Atlas, 1989, 287p.

20. TAKAHASHI, Regina Toshie. Sistema de informação em enfermagem. In: KUR GANT, P. (cood.). Administração em enfermagem. São Paulo: EPU, 1991. p. 181 a 189.

21. WILSON, Holly Skodol. Research In nursing. Califórnia: Addison Wesley Publishing Company, Nursing Division, 1985. 583p. 